\title{
ALTERNATIVAS PARA TRIAGEM NUTRICIONAL EM IDOSOS DOMICILIADOS EM CENÁRIO PANDÊMICO DO COVID-19 UMA REVISÃO INTEGRATIVA DA LITERATURA ${ }^{1}$
}

\author{
ALTERNATIVES FOR NUTRITIONAL SCREENING IN \\ ELDERLY HOUSEHOLDS IN PANDEMIC SCENARIO OF \\ COVID-19 AN INTEGRATIVE LITERATURE REVIEW
}

\author{
Alexander Miranda Pereira ${ }^{2}$, Tereza Cristina Blasi ${ }^{3}$, \\ Thiago Durand Mussoi ${ }^{4}$ e Camila Lehnhart Vargas ${ }^{5}$
}

\section{RESUMO}

Esta revisão buscou identificar um instrumento de triagem nutricional mais adequado para prevenção dos agravos em saúde da pessoa idosa domiciliada a ser utilizado frente a um cenário de pandemia. Trata-se de uma revisão integrativa de literatura, utilizando as bases de dados Scielo, Bireme e PubMed, os critérios de elegibilidade foram: Publicações a partir de 2010, contendo objetivo, metodologia e resultados disponíveis, elegendo pesquisa-ação, pesquisas etnográficas, pesquisa participante, estudos de levantamento, de campo e revisões, contemplando a temática central triagem nutricional em idosos, que considerassem pessoa idosa aquela com 60 anos ou mais, idosos domiciliados, institucionalizados e idosos vinculados/assistidos em Unidades Básicas de Saúde. Foram exclusos: Estudos com idosos hospitalizados, adultos ou crianças, que não usaram instrumento de triagem nutricional, publicados em outro idioma que não inglês, português ou espanhol, pesquisas com animais, in vitro, protocolos, guidelines, diretrizes, cartas e recomendações. Foram analisados os instrumentos de triagem dos estudos e se houve contato físico ou não para aplicação do instrumento, posteriormente buscou identificar o instrumento de triagem nutricional mais adequado. A busca resultou em 362 artigos, excluídos 333 artigos por título, cinco excluídos por serem repetidos, 18 estudos foram excluídos por população estudo e cenário, restando seis que estavam de acordo com os critérios de elegibilidade. Em último momento foi destacado apenas 1 instrumento que correspondeu aos requisitos da pesquisa. $\mathrm{O}$ instrumento NSI apresentou-se mais adequado para rastreio do risco nutricional em idosos domiciliados. Vale ressaltar que o instrumento demanda mais pesquisas para determinar a sua adequação para o público-alvo.

Palavras-chave: Avaliação nutricional, Atenção Primária à Saúde, Geriatria.

\section{ABSTRACT}

This review sought to identify a more appropriate nutritional screening instrument for the prevention of health problems in the elderly household to be used in a pandemic scenario. This is an integrative literature review, using the Scielo, Bireme and PubMed databases, the eligibility criteria were: Publications from 2010, containing objective, methodology and available results, choosing action research, ethnographic research, research participant, survey, field and review studies, contemplating the central theme of nutritional

1 Trabalho Final de Graduação (TFG) do Curso de Nutrição - Universidade Franciscana (UFN).

2 Autor. Bacharel em Nutrição e Pós-graduando em Terapia intensiva com ênfase em oncologia e controle de infecção hospitalar - Universidade Franciscana, Pós-graduando em Bioquímica e Fisiologia de Nutrição-Universidade de São Caetano do Sul. E-mail: alexandermmpp@gmail.com

3 Coautor. Docente do curso de Nutrição - Universidade Franciscana. tcb29@hotmail.com

4 Colaborador. Docente do curso de Nutrição - Universidade Franciscana (UFN). E-mail: tdmussoi@gmail.com

5 Orientador. Docente do Curso de Nutrição - Universidade Franciscana (UFN). E-mail: camila.lehnhart@prof.ufn. edu.br 
screening in the elderly, who considered elderly people to be those aged 60 years or more, elderly people living in the home, institutionalized and elderly linked/assisted in Basic Health Units. Excluded were: Studies with hospitalized elderly, adults or children, who did not use a nutritional screening instrument, published in a language other than English, Portuguese or Spanish, animal research, in vitro, protocols, guidelines, guidelines, letters and recommendations. The screening instruments of the studies were analyzed and whether there was physical contact or not for the application of the instrument, later we sought to identify the most appropriate nutritional screening instrument. The search resulted in 362 articles, 333 articles excluded by title, five excluded for being repeated, 18 studies were excluded by study population and setting, leaving six that met the eligibility criteria. At the last moment, only 1 instrument was highlighted that corresponded to the research requirements. The NSI instrument was more suitable for screening nutritional risk in elderly people living in the home. It is noteworthy that the instrument requires further research to determine its suitability for the target audience.

Keywords: Geriatrics, Nutrition Assessment, Primary Health Care.

\section{INTRODUÇÃO}

Todo ser humano a partir da sua concepção intrauterina está em constante transformação e adaptação, isso transcende por toda a vida, porém em decorrência do avanço da idade cronológica e biológica o organismo humano é acometido pela senescência, a qual é caracterizada como processo de envelhecimento que por sua vez, engloba determinadas alterações que elucidam fragilidades, esse contexto torna o indivíduo sensível às alterações no seu estado de saúde (MALAGUTTI, BERGO, 2010).

O grupo que convive com o resultado destas modificações são os idosos, que segundo o Estatuto do Idoso é toda pessoa que tem 60 anos de idade ou mais, a partir desta idade é considerado o princípio da elucidação das fragilidades resultantes da adaptação fisiológica do avanço da idade (BRASIL, 2013). Esta faixa etária é representada por 125 milhões de pessoas com 80 anos ou mais, a expectativa de vida aumentou, sendo que pela primeira vez na história, espera-se viver 60 anos ou mais, diante deste cenário projeta-se que no ano de 2050, a população mundial com 60 anos ou mais chegue a 2 bilhões, em contraponto aos 900 milhões em 2015 (BRASIL, 2018). Junto ao aumento da população idosa, a procura de assistência em saúde irá subir, tornando necessário a elaboração de estratégias de promoção e controle de saúde, para que assim consiga promover uma assistência adequada para esta faixa etária (VERAS, 2012).

O processo do declínio das funções fisiológicas resulta em complicações psicológicas e sociais, uso de medicações, dependência funcional e dificuldade na alimentação, aumentando a probabilidade de doenças crônicas (MORAES et al., 2008). Esta condição do idoso torna constante as alterações nutricionais, e assim surge a demanda de uma avaliação ampla e interdisciplinar, sendo imprescindível acompanhar o estado nutricional para que diminua o impacto das alterações nutricionais decorrentes do processo de envelhecimento (SBGG, 2011).

A avaliação nutricional engloba diferentes métodos e técnicas com o objetivo de identificar a presença de distúrbios nutricionais, viabilizando a elaboração de intervenções adequadas e precoces, 
promovendo recuperação e manutenção da saúde de um indivíduo. Os métodos constituintes da avaliação nutricional são, Triagem nutricional, Exame clínico, avaliação antropométrica, Composição corporal e exames bioquímicos (MALAGUTTI, BERGO, 2010).

$\mathrm{Na}$ avaliação nutricional da pessoa idosa, cada item da avaliação nutricional representará um indicativo de alteração devido ao processo de senescência, sendo que os inquéritos alimentares demonstraram se há desequilíbrio quanto a ingestão alimentar e necessidade, avaliação clínica, bioquímica e antropométrica indicaram alteração na reserva corporal, diminuição da capacidade funcional devido a carências nutricionais e resposta metabólica quanto a ingestão alimentar (CAMPOS et al., 2007).

Logo a Triagem nutricional tanto no adulto como no idoso, almeja englobar todos estes aspectos abordados anteriormente, porém de forma subjetiva, sucinta, com baixo custo e ágil, tendo o objetivo determinar o grau de risco nutricional que o indivíduo está sendo um instrumento que permite direcionar indivíduos com risco nutricional mais elevado para uma avaliação nutricional mais detalhada para que haja intervenções precisas e profiláticas (MARTINS, 2008).

Atualmente vivemos em um cenário pandêmico, o qual teve origem em dezembro de 2019 por meio de casos de pneumonia grave, posteriormente em achados clínicos foi descoberto que a SARS-CoV-2 foi o causador dos casos de pneumonia grave, sendo esse o agende causador da covid-19 que atingiu nível mundial (ESTEVÃO, 2020). Esta condição é um desafio para todas as áreas de atuação, até mesmo para os profissionais da saúde, onde a demanda para criação de estratégias e reorganização na assistência é alta (HAMMERSCHMIDT, SANTANA, 2020). Neste contexto a população idosa é classificada como grupo de risco devido a imunossenescência, condição que torna o indivíduo vulnerável a doenças infectocontagiosas, porém deve ser mantida as medidas de promoção à saúde da pessoa idosa, para isso os profissionais devem elaborar metodologias de atendimento considerando as medidas preventivas de contágio do COVID-19 (MEDINA et al., 2020).

Conforme os autores supracitados, o estado nutricional da pessoa idosa é um aspecto determinante para prevenção de agravos em saúde decorrentes do processo de envelhecimento. Os instrumentos de triagem nutricional permitem a identificação precoce de risco nutricional possibilitando à equipe de saúde agir de forma profilática, reduzindo declínios funcionais da pessoa idosa. Tendo em vista o cenário que vivemos no Brasil e no mundo pela COVID-19, é necessário buscar ferramentas para realizar o acompanhamento do estado nutricional da população idosa, sem intervenções desnecessárias, na busca de menor contato físico, em especial no trabalho preventivo realizado na atenção primária à saúde.

A importância da identificação do risco nutricional em idosos, por diferentes métodos de avaliação é objeto de diferentes estudos, sendo assim, o objetivo desta revisão integrativa da literatura é identificar qual seria o melhor instrumento de triagem nutricional na prevenção aos agravos em saúde da pessoa idosa a ser utilizado frente ao cenário de pandemia em idosos domiciliados. 


\section{METODOLOGIA}

Este estudo é caracterizado como um estudo de revisão de literatura, do tipo revisão integrativa. Este método permite uma análise ampla de literatura, possibilitando discussões sobre métodos e resultados de pesquisas, sendo assim esta estrutura de pesquisa constitui um profundo entendimento de determinado fenômeno baseando-se em estudos anteriores (WHITTEMORE, KNAFL, 2005).

Para elaboração da revisão integrativa em questão foi seguido determinadas etapas, das quais: Identificação do tema e seleção da hipótese ou questão de pesquisa para a elaboração da revisão integrativa. Estabelecimento de critérios para inclusão e exclusão de estudos/amostragem ou busca na literatura. Definição das informações a serem extraídas dos estudos selecionados/categorização dos estudos. Avaliação dos estudos incluídos na revisão integrativa. Interpretação dos resultados. Apresentação da revisão/síntese do conhecimento. Esta proposta otimiza a síntese de conhecimento devido sua sequência lógica e organizada (MENDES, SILVEIRA, GALVÃO, 2008).

Para determinação dos descritores foi feita uma consulta no Descritores em Ciências da Saúde (DeCS), considerando os descritores: No português, Nutricional, Triagem, Instrumentos, Idoso. inglês: Nutritional; Screening; Instruments; Elderly. No espanhol: Nutricional, Criabado, Instrumentos, Anciano, na busca foi utilizado os boleanos OR, AND e NOT para viabilizar a maior captação de estudos sobre o respectivo tema. A busca de dados foi realizada nas plataformas Pudmed, Bireme e Scielo, no período de setembro a outubro de 2020.

Considerou-se como critério de elegibilidade para revisão, artigo publicados a partir de 2010, com no mínimo objetivo, metodologia e resultados disponíveis, sendo considerado estudos de pesquisa-ação, pesquisas etnográficas, pesquisa participante, estudos de levantamento, de campo e revisões, contemplando a temática central triagem nutricional em idosos, os quais consideravam pessoa idosa aquela com 60 anos ou mais, considerando idosos domiciliados, institucionalizados e idosos vinculados/assistidos em Unidades Básicas de Sáude (UBS).

Foram excluídos todos aqueles estudos com triagem nutricional em idosos hospitalizados, com adultos ou crianças, que não usaram instrumento de triagem nutricional, publicados em outro idioma que não inglês, português ou espanhol. Também desconsiderado para esta revisão pesquisas com animais, in vitro, protocolos, guidelines, diretrizes, cartas e recomendações.

Posteriormente a coleta de dados conforme descrito anteriormente, foi realizada a pré-seleção dos artigos por meio da leitura criteriosa dos títulos e resumos, no intuito de verificar a adequação deles aos critérios estabelecidos. Dos seis estudos por fim selecionados nesta revisão, foram destacados e armazenados os seguintes itens em uma tabela no Microsft Word: Objetivo, metodologia e instrumento de triagem nutricional utilizados, bem como os resultados encontrados pelos autores das pesquisas.

Em último momento, foi identificado se houve contato físico direto com os participantes, como; fazer aferições de circunferências, dobras cutâneas, peso e altura para avaliação do risco 
nutricional com o tipo de instrumento de triagem nutricional utilizado. Para tal identificação julgou-se o instrumento de triagem mais adequado para uso na população idosa domiciliada frente a pandemia do COVID-19, aquele instrumento de triagem nutricional que: Possa ser aplicado presencialmente com distanciamento físico, via conferência online ou apenas por autopreenchimento dos idosos e/ou familiares, considere apenas informações de autorrelato, seja validado para aplicar em idosos domiciliados, tenha exclusividade para identificação de risco nutricional em idosos, e avalie no mínimo aspectos antropométricos, dietéticos, socioeconômicos e capacidade funcional.

Posteriormente foi feita a interpretação dos estudos selecionados, pôr fim a identificação do método de triagem que contemplou os requisitos do objetivo do estudo, o qual é avaliar o risco nutricional da pessoa idosa brasileira sem contato físico direto.

\section{RESULTADOS}

A partir da busca nos bancos de dados com os respectivos descritores pré-estabelecidos foram encontrados 362 artigos. Foram excluídos 333 artigos por título, pois estes não estavam de acordo com os requisitos estabelecidos na revisão em questão, tratando-se de pesquisas com assuntos divergentes com a busca desta revisão. Destes, cinco estudos estavam repetidos nos bancos de dados, por fim foi feita a leitura completa dos 24 estudos restantes, sendo excluídos 18 pois apresentavam pesquisas em cenário e grupo não condizentes com o objetivo deste estudo. Restou seis pesquisas, estas estavam de acordo com os critérios de elegibilidade exposto na metodologia desta revisão (Figura 1).

Figura 1 - Organograma da seleção dos estudos que utilizaram instrumentos de triagem nutricional em idosos domiciliados.

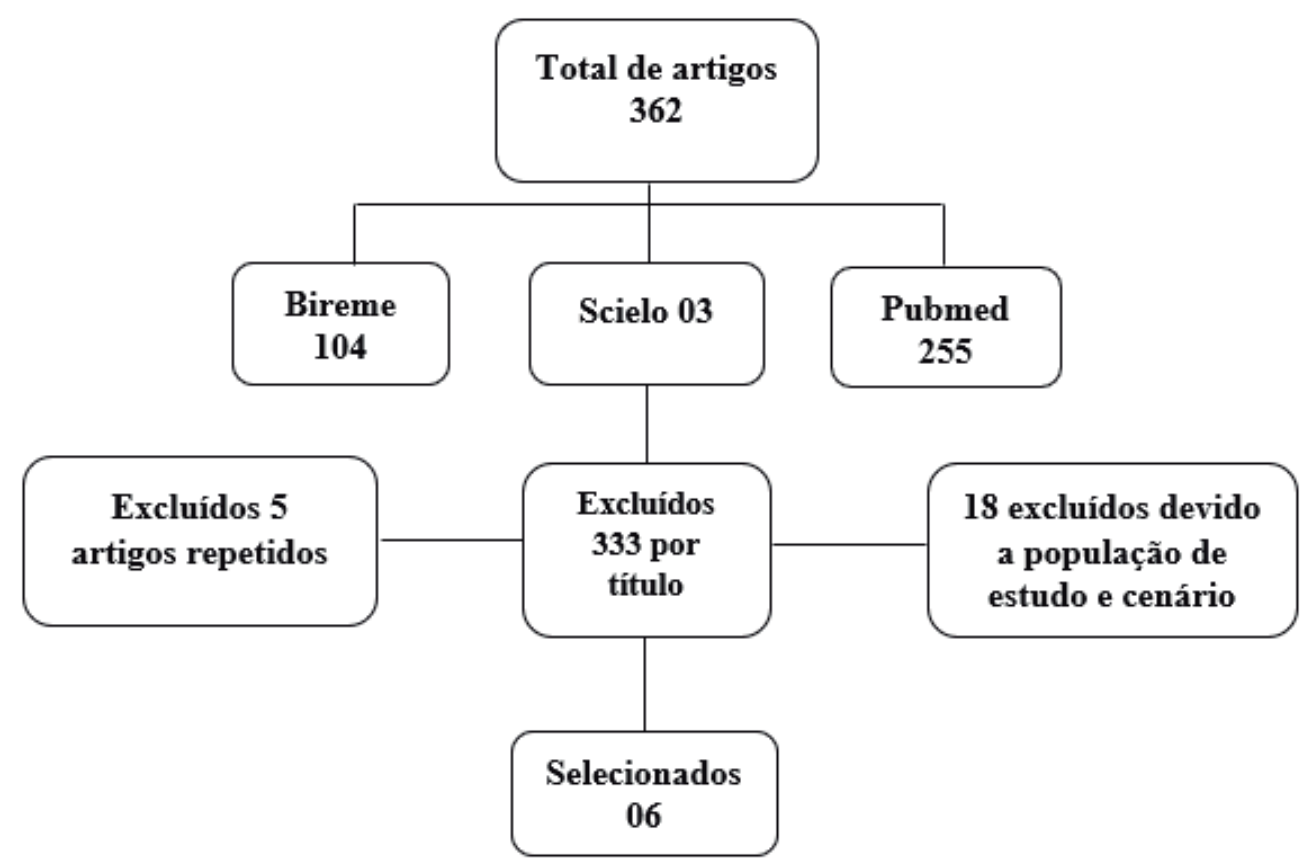

Fonte: MIRANDA et al. (2020). 
Dos estudos selecionados (Quadro 1) apenas um artigo aplicou instrumento de triagem nutricional em idosos brasileiros domiciliados, o restante na Noruega, Nepal, República Tcheca e Eslovaca. Os instrumentos aplicados nas pesquisas selecionadas, foram a Mini Avaliação nutricional (MNA), Mini Avaliação Nutricional-ShortForm (MNA-SF), Nutritional Form For the Elderly (NUFFE), Avaliação subjetiva Global(ASG),Malnutrition Universal Screening Tool(MUST), Nutrition screening initiative (NSI). Dos instrumentos identificados, NUFFE e a MNA se repetiram em estudos diferentes, sendo cada uma utilizada em três estudos e aplicadas com o mesmo público (idosos domiciliados).

Em relação a aplicabilidade sem contato físico, 4 estudos utilizaram instrumentos de triagem nutricional que não tinham contato físico, apenas informações autorrelatadas por idosos. Destes estudos 3 eram com o instrumento NUFFE e um com o NSI. Mesmo que avaliado apenas com informações de autorrelato, os estudos demonstraram a classificação de risco nutricional do público-alvo, sendo que especificamente o NSI foi aplicado na população idosa brasileira em domicílio.

Quadro 1 - Caracterização dos estudos que utilizaram instrumentos de triagem nutricional em idosos domiciliados.

\begin{tabular}{|c|c|c|c|c|}
\hline Autor; Ano; País & Objetivo & Metodologia & $\begin{array}{c}\text { Instrumento de triagem } \\
\text { utilizado }\end{array}$ & $\begin{array}{l}\text { Houve } \\
\text { contato } \\
\text { físico? }\end{array}$ \\
\hline $\begin{array}{l}\text { GHIMIRE, } \\
\text { BARAL, } \\
\text { CALLAHAN, } 2017 . \\
\text { Nepal }\end{array}$ & $\begin{array}{l}\text { Avaliar o estado nutricional da } \\
\text { população idosa em uma aldeia } \\
\text { típica de Okharpauwa. } \\
\text { Validar o instrumento Mini } \\
\text { Avaliação Nutricional (MNA). }\end{array}$ & $\begin{array}{l}\text { Estudo Transversal com } 242 \\
\text { Idosos de } 69 \text { anos residentes } \\
\text { em Okharpauwa }\end{array}$ & $\begin{array}{l}\text { MNA: } \\
\text { Aspectos subjetivos; Peso; } \\
\text { Altura; } \\
\text { Percentual de Perda de peso; } \\
\text { circunferência do braço e } \\
\text { panturrilha }\end{array}$ & Sim \\
\hline $\begin{array}{l}\text { KOZAKOVA, } \\
\text { JAROSOVA, } \\
\text { ZELINICOVA, } \\
2011 . \\
\text { República Tcheca e } \\
\text { Eslovaca }\end{array}$ & $\begin{array}{l}\text { Comparar três instrumentos de } \\
\text { triagem e avaliação do estado } \\
\text { nutricional de idosos em suas } \\
\text { residências. }\end{array}$ & $\begin{array}{l}\text { Teste de instrumentos de me- } \\
\text { dição; } 120 \text { idosos residentes na } \\
\text { República Tcheca e Eslovaca } \\
\text { com } 65 \text { anos ou mais. }\end{array}$ & $\begin{array}{l}\text { MNA: } \\
\text { Aspectos subjetivos; Peso; } \\
\text { Altura } \\
\text { Percentual de Perda de peso; } \\
\text { circunferência da panturrilha } \\
\text { e braço. } \\
\text { ASG: } \\
\text { Aspectos subjetivos; Peso; } \\
\text { Altura } \\
\text { Percentual de Perda de peso. } \\
\text { MUST: } \\
\text { Aspectos subjetivos; Peso; } \\
\text { Altura; } \\
\text { Percentual de Perda de peso. }\end{array}$ & Sim \\
\hline $\begin{array}{l}\text { SODERHAMN } \\
\text { et al., } 2012 . \\
\text { Noruega }\end{array}$ & $\begin{array}{l}\text { Investigar a prevalência de } \\
\text { risco nutricional em idosos } \\
\text { domiciliados e identificar } \\
\text { fatores associados usando dois } \\
\text { instrumentos diferentes de } \\
\text { triagem nutricional. }\end{array}$ & $\begin{array}{l}\text { Transversal; } 2106 \text { idosos com } \\
65 \text { anos ou mais domiciliados. }\end{array}$ & $\begin{array}{l}\text { MNA-SF } \\
\text { Aspectos subjetivos; Peso; } \\
\text { Altura; IMC. } \\
\text { NUFFE: } \\
\text { Informações autorrelatadas } \\
\text { sobre capacidade funcional, } \\
\text { socioeconômica e consumo } \\
\text { alimentar. }\end{array}$ & Não \\
\hline
\end{tabular}




\begin{tabular}{|c|c|c|c|c|}
\hline $\begin{array}{l}\text { SODERHAN } \\
\text { et al., } 2010 . \\
\text { Noruega }\end{array}$ & $\begin{array}{l}\text { Identificar os fatores para risco } \\
\text { nutricional, por meio de uma } \\
\text { triagem nutricional, em uma } \\
\text { amostra de base populacional } \\
\text { de pessoas com } 75 \text { anos de ida- } \\
\text { de residentes em três conselhos } \\
\text { municipais na Suécia. }\end{array}$ & $\begin{array}{l}\text { Transversal; } 1461 \text { idosos com } \\
75 \text { anos residentes em três con- } \\
\text { selhos municipais na Suécia. }\end{array}$ & $\begin{array}{l}\text { NUFFE: } \\
\text { Informações autorrelatadas } \\
\text { sobre capacidade funcional, } \\
\text { socioeconômica e consumo } \\
\text { alimentar. }\end{array}$ & Não \\
\hline $\begin{array}{l}\text { ALVARENGA } \\
\text { et al., } 2010 . \\
\text { Brasil }\end{array}$ & $\begin{array}{l}\text { Descrever o perfil sociode- } \\
\text { mográfico e as condições de } \\
\text { saúde de idosos atendidos por } \\
\text { Equipes de Saúde da Família } \\
\text { no município de Dourados, } \\
\text { MS, avaliar o risco nutricional } \\
\text { por meio do NSI e verificar a } \\
\text { associação entre ambos. }\end{array}$ & $\begin{array}{l}\text { Transversal; } 503 \text { Idosos com } \\
60 \text { anos ou mais residentes em } \\
\text { Dourados-MS. }\end{array}$ & $\begin{array}{l}\text { NSI: } \\
\text { Informações autorrelatadas } \\
\text { sobre aspectos dietéticos antro- } \\
\text { pométricos socioeconômicos e } \\
\text { capacidade funcional. }\end{array}$ & Não \\
\hline $\begin{array}{l}\text { TOMSTAD } \\
\text { et al.,2012. } \\
\text { Noruega }\end{array}$ & $\begin{array}{l}\text { Descrever a prevalência de } \\
\text { risco de desnutrição entre um } \\
\text { grupo de idosos residentes em } \\
\text { casa na Noruega e relacionar } \\
\text { os resultados com a capacidade } \\
\text { relatada de autocuidado, senso } \\
\text { de coerência, percepção de } \\
\text { saúde e outras questões relacio- } \\
\text { nadas à saúde. }\end{array}$ & $\begin{array}{l}\text { Transversal; } 450 \text { idosos com } 65 \\
\text { anos ou mais residentes no sul } \\
\text { da Noruega. }\end{array}$ & $\begin{array}{l}\text { NUFFE: } \\
\text { Informações autorrelatadas } \\
\text { sobre capacidade funcional, } \\
\text { socioeconômica e consumo } \\
\text { alimentar. }\end{array}$ & Não \\
\hline
\end{tabular}

Fonte: Miranda et al., (2020)

\section{DISCUSSÃO}

A cartilha elaborada pelo Conselho Federal de Nutricionistas (CFN, 2020), recomenda que tanto na atenção básica como em outras áreas de cuidados, os nutricionistas devem analisar o cenário e as normativas locais para entender a gravidade do problema, assim considerar as importantes limitações impostas pela ausência da avaliação e contato físico com o paciente, e assim avaliar a melhor modalidade de atendimento para uma adequada assistência nutricional. Diante desta recomendação, esta revisão destaca um método de triagem nutricional que considera as limitações de contato físico e contempla o rastreio de risco nutricional em idosos domiciliados, conforme será discutido.

Como exposto no quadro 1 , dentre os seis diferentes métodos encontrados, observou-se que apenas dois métodos utilizaram informações autorrelatadas, destes apenas um foi aplicado no Brasil. Os demais instrumentos (MNA, ASG, MUST, MNA-SF) exigem alguma aferição antropométrica (Peso, Altura e/ou circunferências) a que demanda contato físico.

Um estudo comparou três instrumentos de triagem nutricional, dos quais, MNA, ASG e MUST, o comparativo ocorreu em 120 idosos residentes na República Tcheca e Eslovaca. Constatou-se que os métodos mostraram divergência quanto à classificação de risco nutricional, sendo que a MNA classificou 42 idosos com desnutrição; ASG determinou que 11 idosos estavam com desnutrição grave e o MUST apresentou 21 idosos com alto risco para desnutrição. Os métodos levam em 
consideração aspectos subjetivos de autorrelato, mas demandam diferentes parâmetros antropométricas para determinação do risco nutricional como por exemplo: Circunferência do Braço e Panturrilha (MNA), Peso, Altura, Percentual de Perda de Peso (ASG e MUST) (KOZAKOVA, JAROSOVA, ZELINICOVA, 2011).

Uma pesquisa de validação da MNA para triagem de risco nutricional em idosos residentes na zona rural do Nepal, Ghimire, Baral e Callahan, (2017) utilizaram e validaram o instrumento considerando as aferições antropométricas (Peso, Altura, Circunferência do braço e panturrilha) para rastreio do risco nutricional. Soderhamn et al.,(2012) que comparou dois instrumento de triagem nutricional, ressalta que a MNA e MNA-SF são recomendadas pelas European Society for Clinical Nutrition and Metabolism (ESPEN) para rastreio nutricional, porém cita que as aferições antropométricas consideradas pela MNA inviabilizam o rastreio de risco nutricional por autorrelato, assim o autor justifica o uso da versão MNA-SF pois mesmo que este instrumento considere o IMC como fator de avaliação ele pode ser aplicado com informações autorrelatadas. Neste mesmo estudo de Soderhamn et al., (2012) foi comparado a MNA-SF com o NUFFE o qual identifica o risco nutricional por meio de 15 questões de autorrelato, os autores verificaram que o NUFFE classificou mais indivíduos com risco de desnutrição, por fim ambos os instrumentos têm efetividade para rastrear risco nutricional em idosos domiciliados, porém o NUFFE classifica precocemente o idoso com risco nutricional.

O NUFFE é utilizado em outro estudo para rastrear os fatores de risco para risco nutricional em idosos por meio de um instrumento de triagem. Este instrumento foi escolhido pois possibilita a avaliação de um grande número de idosos por ser um instrumento de autorrelato. O pesquisador enviou por correio o instrumento para 1461 idosos com 75 anos, posteriormente com a devolutiva foi identificado que 77,7\% dos estudados apresentavam baixo risco nutricional segundo o NUFFE, os autores concluíram que o instrumento possibilitou a identificação de fatores de risco nutricional e ainda ressalta que o instrumento é de fácil aplicação e viabiliza o rastreio nutricional (SODERHAMN et al., 2010).

Outro trabalho desenvolvido na Noruega com idosos de 65 anos ou mais também utilizou o instrumento de triagem nutricional NUFFE para rastrear risco nutricional, no estudo o instrumento apontou que a maior parte dos participantes apresentavam baixo risco de desnutrição (79,7\%). Os autores concluíram a triagem dos participantes com informações autorrelatadas, pois o instrumento foi enviado por correio para que os participantes preenchessem, e não houve outro método ou parâmetro antropométrico para rastreio (TOMSTAD et al., 2012).

No Brasil foi conduzido um estudo transversal com 503 idosos residentes no município de Dourados - MS, este estudo descreveu o perfil sociodemográfico e as condições de saúde de idosos atendidos por Equipes de Saúde da Família, e avaliou o risco nutricional por meio do NSI posteriormente verificou associação entre ambos. Para avaliar o risco nutricional, o autor utilizou o instrumento 
de triagem nutricional NSI. Este instrumento contempla 10 perguntas com informações autorrelatadas sobre aspectos dietéticos antropométricos socioeconômicos e capacidade funcional sendo estas perguntas apenas de autorrelato, sem avaliação de composição corporal. Além do autor a coleta de dados teve auxílio de três acadêmicas do curso de enfermagem, que foram habilitadas através de um curso prático de 20 horas para aplicar o instrumento. Contudo o instrumento forneceu o rastreio de idosos com risco nutricional, e ainda se destacou que este método de triagem possibilita a aplicação na Atenção Básica por agentes comunitários de saúde, pois é um instrumento rápido, fácil e barato de aplicar, que não exige cursos extensos para habilitar profissionais na aplicabilidade do instrumento (ALVARENGA et al., 2010).

Três instrumentos apresentaram eficiência no rastreio de risco nutricional com informações de autorrelato em idosos, porém o NUFFE é um instrumento que foi aplicado apenas em dois países, já a MNA-SF, mesmo que aplicada em apenas um país da pesquisa realizada, é um instrumento recomendado pelas Diretrizes Europeias para rastreio nutricional, já o NSI não é considerado pelas Diretrizes, porém o instrumento foi aplicado em idosos brasileiros e os autores apresentaram que sua aplicabilidade neste cenário foi efetiva em determinar o risco nutricional.

Dos instrumentos de triagem aqui revisados destaca-se até então o NSI devido sua prévia aplicabilidade na população idosa brasileira. Este instrumento foi elaborado por uma força tarefa americana denominada Nutrition screening initiative. Roediger et al., (2017) elaborou uma tradução e adaptação transcultural do instrumento para o português Brasil e ainda adaptou e validou o instrumento para aplicar na população idosa brasileira. Na pesquisa os autores constataram que foram possíveis as adaptações e por fim validaram o NSI para uso em idosos brasileiros domiciliados.

\section{CONCLUSÃO}

Por meio desta revisão foi possível elucidar que o MNA-SF, NUFFE e NSI são instrumentos que não demandam contato físico para rastrear risco nutricional na população idosa domiciliada. Dos instrumentos identificados, o NSI demonstrou-se mais adequado devido a adaptação transcultural validada para população idosa brasileira em domicílio e sua prévia aplicação neste grupo.

Destaca-se que o instrumento NSI considera o contexto de saúde geral da pessoa idosa para determinação do risco nutricional, sendo assim possivelmente possibilita prevenção de agravos em saúde da pessoa idosa, por meio de uma avaliação de autorrelato respeitando as medidas preventivas de contágio do COVID-19.

Conduto esta revisão com base nos estudos encontrados e analisados faz uma proposição para o uso do NSI em idosos domiciliados, considera-se que o instrumento demanda mais pesquisas para realização de uma efetiva recomendação. 


\section{REFERÊNCIAS}

ALVARENGA, M. R. et al. Avaliação do risco nutricional em idosos atendidos por Equipes de Saúde da Família / Evaluation of the nutritional risk in elderly assisted by Family Health Teams / Evaluación del riesgo nutricional en ancianos atendidos por Equipos de Salud de Familia. Revista da Escola de Enfermagem, v. 44, n. 4, p. 1046-1051, Dez, 2010.

BRASIL. Ministério da Saúde. Estatuto do Idoso / Ministério da Saúde - 3. ed., 2. reimpressão. Brasília: Ministério da Saúde, 2013.

BRASIL. Organização Mundial da Saúde. Folha Informativa: Envelhecimento e Saúde, Fev, 2018.

CAMPOS, M. A. et al. Estado Nutricional e Antropometria em Idosos: Revisão da Literatura. Revista Médica de Minas Gerais, v. 17, p. 111-120, 2007.

CONSELHO FEDERAL DE NUTRICIONISTAS (CFN). Recomendações do CFN: Boas práticas para a atuação do nutricionista e do técnico em nutrição e dietética durante a pandemia do novo coronavírus (COVID-19). 3. Ed., Brasília, Mar, 2020.

ESTEVÃO, A. COVID-19. Acta Radiológica Portuguesa, v. 32, n. 1, jan-abr, 2020.

GHIMIRE, S. BARAL, B. CALLAHAN, K. Nutritional Assessment of Community-Dwelling Older Adults in Rural Nepal. PLOS ONE, v. 12, n. 2, Feb, 2017.

HAMMERSCHMIDT, K.S.A. SANTANA, R.F. SAÚDE DO IDOSO EM TEMPOS DE PANDEMIA COVID-19. Cogitare Enfermagem, 2020.

KOZAKOVA, R. JAROSOVA, D. ZELENIKOVA, R. Comparison of three screening tools for nutritional status assessment of the elderly in their homes. Biomed Papers of the Medical Faculty of the University Palacky Olomouc Czech Repub, v. 156, n. 4, p. 371-376, Dec, 2012.

MALAGUTTI, W. BERGO, A. M. Abordagem Interdisciplinar do Idoso, Rio de Janeiro: Livraria e Editora Rubio, 2010.

MARTINS, C. Avaliação do estado nutricional e Diagnóstico. Curitiba: Nutroclinica, 2008. 
MEDINA, Maria Guadalupe et al. Atenção primária à saúde em tempos de COVID-19: o que fazer?.

Cadernos de Saúde Pública, v. 36, n. 8, 2020.

MENDES, K. SILVEIRA, R. GALVÃO, C. Revisão Integrativa: Método de Pesquisa para a Incorporação de Evidências na Saúde e na Enfermagem. Texto e Contexto Enfermagem, v. 17, n. 4, Out-Dez, 2008.

MORAES, E.N. Princípios Básicos de geriatria e gerontologia. Belo Horizonte: Coopmed. 2008.

SOCIEDADE BRASILEIRA DE GERIATRIA E GERONTOLOGIA (SBGG). Consenso Brasileiro de Nutrição e Disfagia em Idosos Hospitalizados. Barueri-SP : Minha Editora, .2011.

SODERHAMN, U. et al. Factors associated with nutritional risk in 75-year-old community living people International Journal of Older People Nursing, v. 7, p. 3-10, Mar, 2012.

SODERHAMN, U. et al. Nutritional screening of older home-dwelling Norwegians: a comparison between two instruments. Clinical Intervention Aging, v. 7, p. 383-391, Set, 2012.

TOMSTAD, S. et al. Living alone, receiving help, helplessness, and inactivity are strongly related to risk of undernutrition among older home-dwelling people. International Journal of General Medicine, v. 5, p. 231-40, Mar, 2012.

VERAS, R. Prevenção de doenças em idosos: os equívocos dos atuais modelos. Cadernos de saúde pública, Rio de Janeiro, v. 28, n. 10, Out. 2012.

WHITTEMORE, R. Knafl, K. The integrative review: update methodology. journal of advanced nursing, 2005. 